\title{
The Potential of sGC Modulators for the Treatment of Age-Related Fibrosis: A Mini-Review
}

\author{
Peter Sandner ${ }^{a, b}$ Peter Berger ${ }^{c}$ Christoph Zenzmaier ${ }^{d}$ \\ a Bayer Pharma AG - Drug Discovery - Cardiology, Wuppertal, and ${ }^{\mathrm{b}}$ Institute of Pharmacology, Hannover Medical \\ School, Hannover, Germany; ' Institute for Biomedical Aging Research, Universität Innsbruck, and d University of \\ Applied Sciences Tyrol, Innsbruck, Austria
}

\section{Key Words}

Extracellular matrix $\cdot$ Proliferation $\cdot$ Myofibroblast .

Transforming growth factor- $\beta \cdot s \mathrm{sGC}$ activators .

sGC stimulators · Nitric oxide · cGMP signaling ·

Phosphodiesterase type $5 \cdot$ Riociguat

\begin{abstract}
Fibrotic diseases cause high rates of morbidity and mortality, and their incidence increases with age. Despite intense research and development efforts, effective and well-tolerated antifibrotic treatments are scarce. Transforming growth factor- $\beta$ signaling, which is widely considered the most important profibrotic factor, causes a pro-oxidant shift in redox homeostasis and a concomitant decrease in nitric oxide (NO) signaling. The NO/cyclic guanosine monophosphate (cGMP) signaling cascade plays a pivotal role in the regulation of cell and organ function in whole-body hemostasis. Increases in $\mathrm{NO} / \mathrm{cGMP}$ can lead to relaxation of smooth muscle cells triggering vasorelaxation. In addition, there is consistent evidence from preclinical in vitro and in vivo models that increased cGMP also exerts antifibrotic effects. However, most of these findings are descriptive and the molecular pathways are still being investigated. Furthermore, in a variety of fibrotic diseases and also during the natural course of aging, $\mathrm{NO} / \mathrm{cGMP}$ production is low, and current treatment approaches to increase cGMP levels might not be sufficient.
\end{abstract}

\section{KARGER}

(C) 2016 S. Karger AG, Basel

E-Mail karger@karger.com

www.karger.com/ger
The introduction of compounds that specifically target and stimulate soluble guanylate cyclase (sGC), the so called sGC stimulators and $\mathrm{sGC}$ activators, might be able to overcome these limitations and could be ideal tools for investigating antifibrotic mechanisms in vitro and in vivo as they may provide effective treatment strategies for fibrotic diseases. These drugs increase cGMP independently from NO via direct modulation of SGC activity, and have synergistic and additive effects to endogenous NO. This review article describes the NO/cGMP signaling pathway and its involvement in fibrotic remodeling. The classes of sGC modulator drugs and their mode of action are described. Finally, the preclinical in vitro and in vivo findings and antifibrotic effects of cGMP elevation via sGC modulation are reviewed. sGC stimulators and activators significantly attenuate tissue fibrosis in a variety of internal organs and in the skin. Moreover, these compounds seem to have multiple intervention sites and may reduce extracellular matrix formation, fibroblast proliferation, and myofibroblast activation. Thus, sGC stimulators and sGC activators may offer an efficacious and tolerable therapy for fibrotic diseases, and clinical trials are currently underway to assess the potential benefit for patients with systemic sclerosis.

(c) 2016 S. Karger AG, Basel

Peter Sandner

Bayer Pharma AG - Drug Discovery - Cardiology

Research Center Wuppertal

DE-42096 Wuppertal (Germany)

E-Mail peter.sandner@bayer.com

Christoph Zenzmaier

University of Applied Sciences Tyrol

Innrain 98

AT-6020 Innsbruck (Austria)

E-Mail christoph.zenzmaier@ fhg-tirol.ac.at 


\section{Fibrosis and Aging}

Approximately $45 \%$ of mortality in Western nations is attributed to fibrotic diseases, characterized by formation of excess fibrous connective tissue in an organ or tissue. Fibrotic diseases encompass organ-specific diseases, such as cardiac fibrosis, idiopathic pulmonary fibrosis, intestinal fibrosis, liver fibrosis and cirrhosis, and multisystem disorders, such as systemic sclerosis, nephrogenic systemic fibrosis, and chronic graft versus host disease. Fibrosis is also observed in the stromal response to many solid tumors. Additionally, fibrosis is one of the key features of vascular remodeling in atherosclerosis and of airway remodeling in patients with asthma [1].

Fibrosis typically develops in response to initial inflammatory processes and the major causes are tissue damage, infection, and autoimmune disease [2]. Aging is considered a risk factor for fibrosis and the incidence increases sharply with advancing age for many fibrosis-associated diseases, e.g. cardiovascular disease and arteriosclerosis, hepatic fibrosis, idiopathic pulmonary fibrosis, renal fibrosis, and benign prostatic hyperplasia [3]. The molecular mechanisms underlying these age-related differences in disease incidence are not well understood. However, several age-related factors have been proposed, including accumulation of cellular or molecular damage, impaired stress response, cellular senescence, dysregulation of telomere biology, loss of proteostasis, and epigenetic alterations $[3,4]$.

Aging is accompanied by increased oxidative stress and accumulation of advanced glycation end products (AGEs) that have both been associated with development of fibrosis suggesting a direct link between oxidative stress and fibrosis in the course of aging $[5,6]$. Furthermore, it has been shown in rat renal fibroblasts that AGEs decreased nitric oxide (NO) production and reduced protein kinase $\mathrm{G}(\mathrm{PKG})$ activity and thus reduced $\mathrm{NO} /$ cyclic guanosine monophosphate (cGMP) signaling that is also decreased in fibrotic remodeling $[1,3,6]$. In consequence to aging, stromal prostate cells secrete elevated levels of inflammatory mediators such as secreted CXC-type chemokines that promote increases in the proliferation rate of epithelial and stromal cells, a hallmark of fibrotic alterations in benign prostatic hyperplasia [7]. It has been demonstrated that cGMP increase is able to attenuate inflammatory response in human prostatic myofibroblasts, suggesting a link between impairment of the NO/cGMP and inflammation-dependent fibrosis [8]. Moreover, in aging mice, decreased Thy- 1 expression in pulmonary fibroblasts was observed, which resulted in the production of a profibrotic extracellular matrix (ECM) [9]. While the severity of fibrosis in response to lung injury is not significantly different in young and aged mice, the latter demonstrate an impaired capacity for fibrosis resolution resulting in persistent fibrosis characterized by the accumulation of senescent and apoptosis-resistant myofibroblasts $[10,11]$.

\section{The Myofibroblast: The Primary Effector Cell in Fibrosis}

Myofibroblasts are the primary effector cells in fibrosis. They are a specialized cell type that combines the ECM-producing characteristics of fibroblasts with the cytoskeletal and contractile properties of smooth muscle cells [12]. Various cell types have been described and discussed as potential precursor cells of myofibroblasts including local epithelial cells, endothelial cells, smooth muscle cells, pericytes, mesenchymal stem cells, and bone marrow-derived fibrocytes. However, it appears likely that local fibroblasts represent the major source of myofibroblasts [12]. ECM stiffness in conjunction with transforming growth factor- $\beta_{1}$ (TGF $\beta 1$ ) represents a primary stimulus for myofibroblast differentiation and persistence in response to tissue damage or chronic inflammation. The differentiated myofibroblasts produce and deposit new ECM components and secrete a variety of growth factors and cytokines, resulting in enhanced proliferation of the remaining fibroblasts. Moreover, myofibroblasts also secrete and activate TGF $\beta 1$, thereby generating an autocrine feed-forward loop that further drives myofibroblast differentiation $[3,12]$.

\section{Molecular Pathways Underlying Myofibroblast Activation and Fibrogenesis}

Soluble factors and cytokines secreted by platelets and infiltrating leukocytes, such as interleukins, tumor necrosis factor, TGF $\beta$, and platelet-derived growth factor (PDGF), play major roles in fibrogenesis. The molecular pathways and complex interplay between initial inflammation, inflammatory cytokines, TGF $\beta 1$, and other growth factors, including PDGF, fibroblast growth factor (FGF), and locally produced cytokines, are not yet fully understood. These mechanisms and relationships may also be age and tissue dependent, and need further investigation. This has led to current treatment approaches that are quite diverse and high failure rates for experi- 
mental medicines in clinical trials. However, there are some common pathways that are central and conserved within age-dependent fibrotic remodeling, such as the above-mentioned myofibroblast differentiation.

\section{The Role of TGF $\beta$ in Fibrosis}

TGF $\beta$ is widely considered the most important profibrotic growth factor and undoubtedly plays a pivotal role in pathogenic fibrogenesis. Consistently, elevated levels of TGF $\beta$ and enhanced TGF $\beta$ signaling have been observed in various fibrotic disorders including renal interstitial fibrosis, idiopathic pulmonary fibrosis, cardiovascular remodeling, chronic liver disease, systemic sclerosis, benign prostatic hyperplasia and tumor-associated reactive stroma $[1,3,13]$. At a cellular level, the profibrotic effects of TGF $\beta$ are mediated via downstream activation of canonical Smad2/3 signaling or via noncanonical Smad-independent activation of mitogen-activated protein kinase (MAPK) and phosphatidylinositol 3-kinase/Akt pathways and result in production and deposition of ECM and secretion of paracrine- and autocrine-acting growth factors, further modulating cellular signaling cascades [1].

TGF $\beta 1$-induced myofibroblast differentiation and myofibroblast-driven formation of ECM components is caused by a pro-oxidant shift in redox homeostasis. The underlying mechanism involves elevation of nicotinamide adenine dinucleotide phosphate oxidase 4-derived hydrogen peroxide and concomitant decreases in reactive oxygen species-scavenging enzymes such as manganesedependent superoxide dismutase (SOD) or the Se-containing enzymes glutathione peroxidase 3 (GPX3) and thioredoxin reductase 1 (TXNRD1) [1, 3, 14]. Of note, fibroblasts from aged mice displayed an impaired capacity to induce the antioxidant response of NFE2-related factor 2, a master regulator of antioxidant genes, following lung injury [10]. The resulting pro-oxidant shift in redox homeostasis leads to NO synthase (NOS) uncoupling and a concomitant decrease in NO signaling and PKG activity $[1,3]$. The first messenger NO triggers the formation of cGMP, which acts as a second messenger and mediates the NO-related effects. Therefore, enhancement of NO/cGMP signaling may target a common pathway in the development of fibrosis and could represent a potential therapeutic strategy to target myofibroblast differentiation in fibrotic diseases. Recently, agents from a specific class of compounds, the so-called soluble guanylate cyclase (sGC) modulators, were approved for cardiopulmonary diseases, and others are in clinical development for cardiovascular indications [15-18]. This class of compounds binds selectively to sGC, thereby stimulating
sGC activity, which causes a significant increase in cGMP levels. As these compounds are effective independent of $\mathrm{NO}$, this could be of importance in fibrotic diseases where endogenous NO production is low. This review summarizes the preclinical evidence for antifibrotic effects of sGC modulators in vitro and in animal models.

\section{The NO/cGMP/Phosphodiesterase Type 5 Signal Transduction Pathway}

One of the most important pathways regulating vascular tone and leading to vasorelaxation is the NO/cGMP/ phosphodiesterase type 5 (PDE5) pathway. This pathway and its pharmacological modulation have been extensively reviewed in the literature and covered in depth in the Handbook of Experimental Pharmacology [19]. In brief, $\mathrm{NO}$ is formed from L-arginine via the activity of NOSs, of which three isozymes have been identified to date: endothelial NOS (eNOS), neuronal NOS, and an inducible form of NOS. In the first step of the pathway, NO formed via NOS is liberated by nerve fibers or endothelial cells and then binds to sGC, which triggers enhanced cGMP formation. In the next step, cGMP regulates the activity of various downstream targets, mainly cGMP-regulated protein kinases (e.g. PKG), cGMP-regulated PDEs, and cGMP-regulated ion channels. These cGMP downstream targets translate the increase in cGMP levels into a decrease in intracellular free calcium levels in smooth muscle cells. Thus, one of the most prominent physiological responses of increasing intracellular cGMP levels, especially in smooth muscle cells, is relaxation of smooth muscle cells. The relaxing effects of cGMP are abrogated by cleavage of cGMP through highly effective PDEs, in particular PDE5 (fig. 1) [19].

\section{Pharmacological cGMP Increase: Modulating sGC Activity}

As the NO/cGMP/PDE5 signaling cascade is of paramount importance for regulation of vascular tone, even before the signal transduction pathway was fully understood, pharmaceuticals were introduced that enhanced vasorelaxation, aiming to improve local blood flow and oxygen supply in tissues [19]. The first pharmacological interventions in this pathway were nitrates and NO donors, which mimic the action of NO, for the treatment of angina pectoris as these compounds effectively dilate coronary blood vessels. More recently, PDE5 inhibitors were 


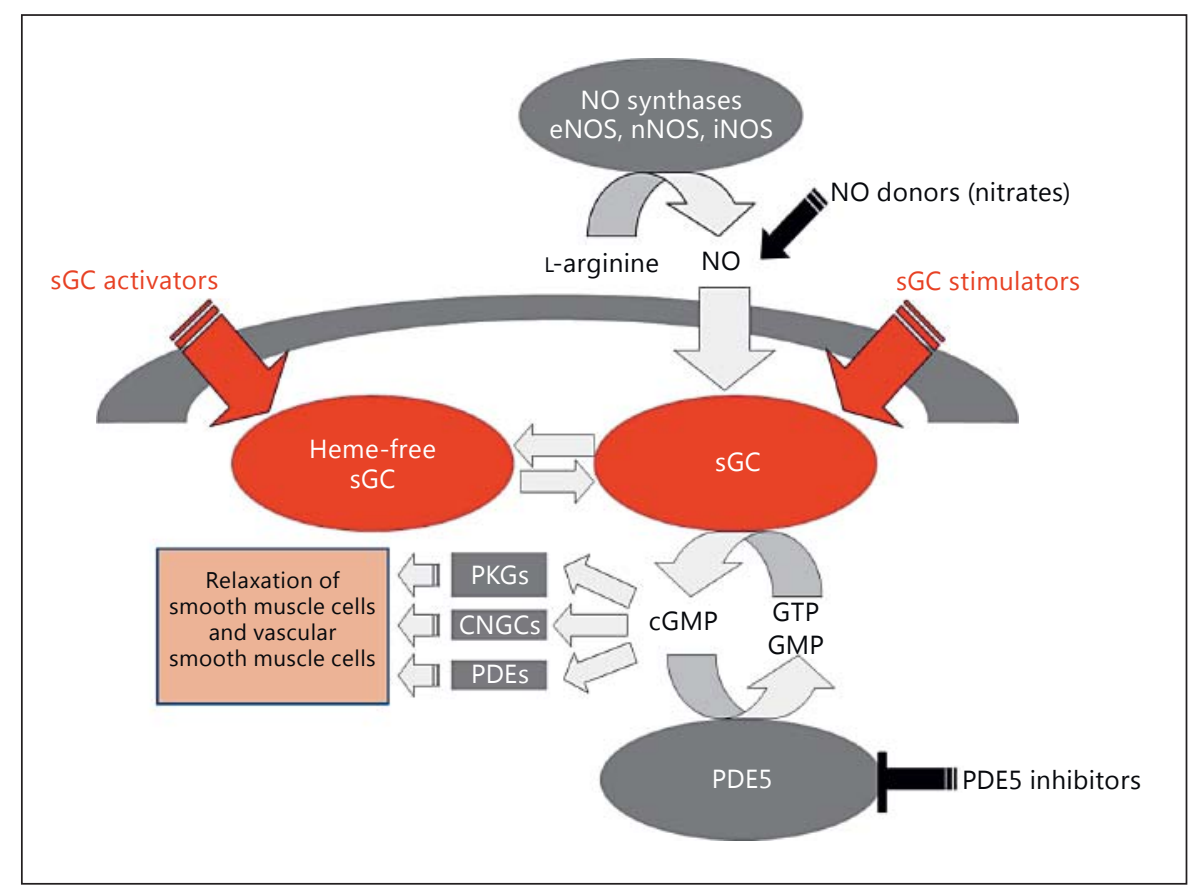

Fig. 1. The NO/cGMP/PDE5 pathway with cGMP downstream targets, cGMP effects, and major pharmacological intervention sites. NO is formed from L-arginine by NO synthases and diffuses into smooth muscle cells and vascular smooth muscle cells where it stimulates sGC, triggering cGMP formation. cGMP acts via different downstream targets and thereby causes decreases in intracellular free calcium levels, resulting in relaxation of smooth muscle cells and vascular smooth muscle cells. NO donors, PDE5 inhibitors, and sGC stimulators lead to an increase in cGMP levels by replacing $\mathrm{NO}$ (nitrates) by blockade of cGMP breakdown (PDE5 inhibitors) and by sGC (sGC stimulation or activation [20, 21]. cGMP, cyclic guanosine monophosphate; CNGC, cyclic nucleotide gated calcium channel; eNOS, endothelial nitric oxide synthase; GMP, guanosine monophosphate; GTP, guanosine triphosphate; iNOS, inducible nitric oxide synthase; $\mathrm{nNOS}$, neuronal nitric oxide synthase; NO, nitric oxide; PDE, phosphodiesterase; PDE5, phosphodiesterase type 5; PKG, protein kinase G; sGC, soluble guanylate cyclase. introduced for the treatment of erectile dysfunction. Some of these compounds were subsequently approved for the treatment of pulmonary arterial hypertension (PAH) and for signs and symptoms of benign prostatic hyperplasia [19].

The most recently introduced class of compounds to effectively target this pathway are modulators of the sGC, the sGC stimulators and sGC activators, which both stimulate sGC independently from NO to trigger increases in cGMP levels. The discovery of these compounds in the late 1990s was a milestone in overcoming the treatment limitations of nitrates and PDE5 inhibitors. Initially, these compounds were termed sGC stimulators and/or sGC activators or compounds stimulating and/or activating $\mathrm{sGC}$, irrespective of their distinct properties with respect to binding to sGC, i.e. heme-containing or hemefree forms of the enzyme. In recent years, two classes of compounds have been defined: sGC stimulators [20] and sGC activators [21].

\section{sGC Stimulators}

sGC stimulators act independently from endogenous $\mathrm{NO} / \mathrm{cGMP}$ production and are able to increase intracellular cGMP levels by direct stimulation of sGC (fig. 1). These compounds act independently from NO, but require the heme group of sGC to be active. The group of sGC stimulators includes YC-1, A-344905, A-350619 and A-778935 from Abbott, and BAY 41-2272, BAY 41-8543, BAY 60-4552 (nelociguat) and BAY 63-2521 (riociguat) from Bayer (fig. 2) [22, 23]. Riociguat was approved in 2013 for the treatment of PAH and chronic thromboembolic pulmonary hypertension (CTEPH) and is marketed as Adempas ${ }^{\circledR}$. Owing to their biochemical properties, these compounds are now termed $\mathrm{NO}$-independent heme-dependent sGC stimulators. They stimulate sGC independently from $\mathrm{NO}$ but also act synergistically with $\mathrm{NO}$ and are able to substantially enhance the effects of very low levels of endogenous NO. 


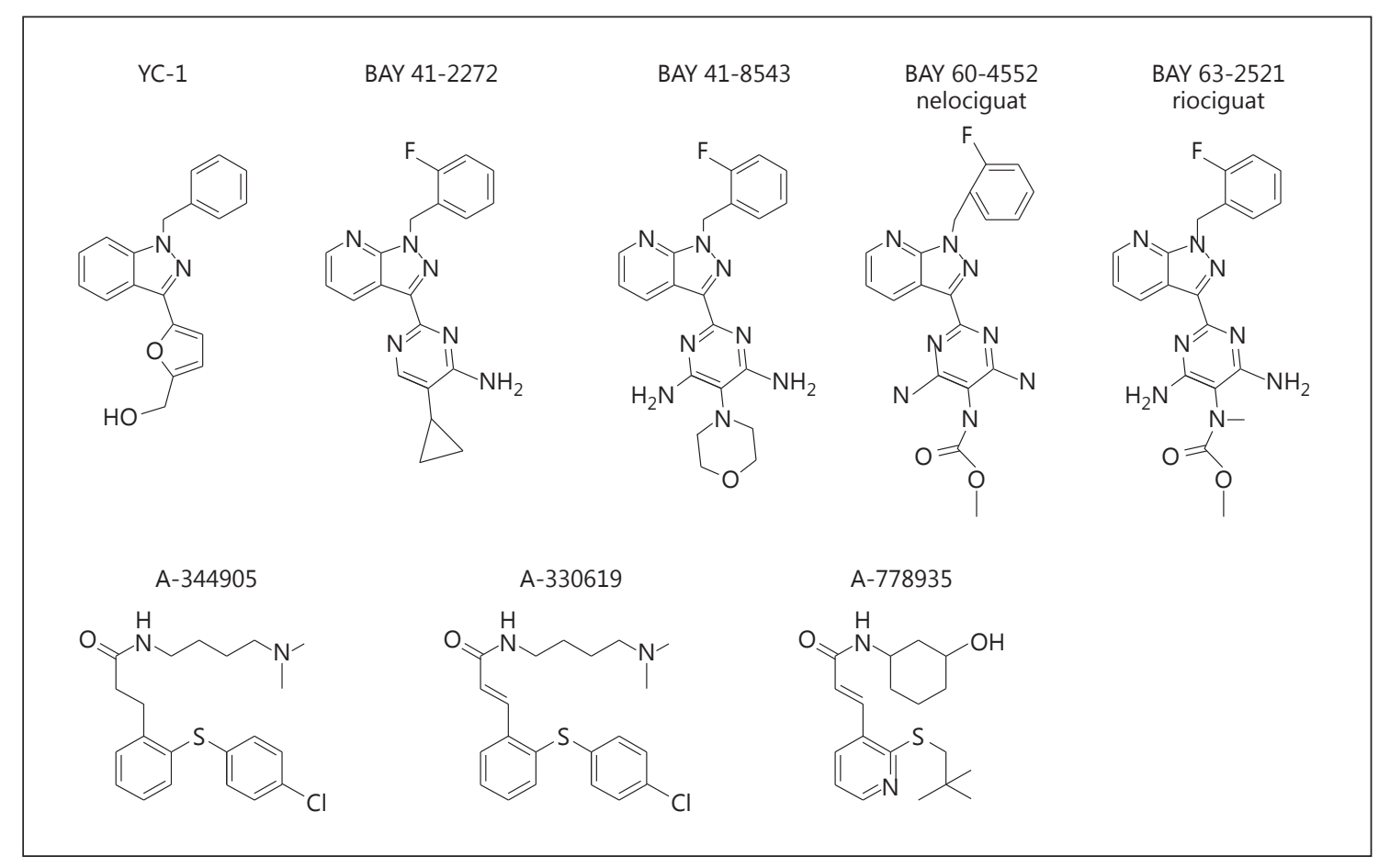

Fig. 2. Chemical structures of NO-independent and heme-dependent sGC stimulators that act synergistically with endogenous NO (see $[22,23])$. The first described sGC stimulator was the benzyl indazole compound YC-1. Replacement of the benzyl indazole moiety of YC-1 by a (2-fluorobenzyl)pyrazolopyridine moiety and subsequent modifications led to the discovery of additional sGC

\section{sGC Activators}

More recently, another group of compounds was developed that activates sGC independently from $\mathrm{NO}$ and does not require the heme group of the enzyme. Thus, these compounds have been termed NO-independent heme-independent sGC activators. This group of compounds includes BAY 58-2667 (cinaciguat) and BAY 602770 from Bayer, and S-3448 and HMR-1766 (ataciguat) from former Hoechst-Marion Roussel (now Sanofi Aventis) (fig. 3) [22,23]. The efficacy of sGC activators is independent from bioavailable NO. Additionally, sGC activators show an additive effect with endogenous NO.

\section{Therapeutic Potential of sGC Stimulators and sGC Activators in Fibrotic Diseases}

Given the broad impact of the NO/cGMP/PDE5 signaling cascade in health and disease, sGC stimulators and activators exhibit broad treatment potential, particularly in the cardiovascular, cardiopulmonary, and cardiorenal stimulators by Bayer (BAY 41-2272, BAY 41-8534, BAY 60-4552, and BAY 63-2521). A novel class of sGC stimulators based on ortho-sulfanyl-substituted cinnamyl amides that are not structurally related to YC-1 was developed by Abbott (A-344905, A-350619, and A-778935). NO, nitric oxide; sGC, soluble guanylate cyclase.

systems. This is indicated by the approval of the sGC stimulator riociguat for $\mathrm{PAH}$ and $\mathrm{CTEPH}$, but also by the ongoing clinical development of the sGC stimulator vericiguat (BAY 1021189) in heart failure $[18,24]$ and research and development efforts with sGC stimulators and activators in chronic kidney diseases [25]. This already demonstrates that the beneficial effects of sGC 'modulators' are much broader than vasodilation alone, which is corroborated by the preclinical literature $[15,26]$. It has been shown that increased cGMP levels from sGC stimulators and sGC activators lead to antifibrotic and antiremodeling effects. In addition, antiproliferative, proapoptotic, and anti-inflammatory effects have been demonstrated influencing epithelial function, angiogenesis, adipocyte differentiation and neuroprotection $[15,25$, 26]. These properties render sGC modulators promising treatment options for fibrotic disorders, especially in the aging population. Clinical trials are already underway to assess the clinical benefit of sGC stimulators, e.g. in patients with skin fibrosis and systemic sclerosis (NCT02283762). 
Fig. 3. Chemical structures of NO-independent and heme-independent sGC activators (see $[22,23])$. Structures of the anthranilic acid derivatives S-3448 and HMR1766, which are sGC activators developed by Hoechst-Marion Roussel, and of the sGC activator BAY 58-2667, an amino dicarboxylic acid identified by Bayer via high-throughput screening, and its derivative BAY 60-2770. NO, nitric oxide; sGC, soluble guanylate cyclase.

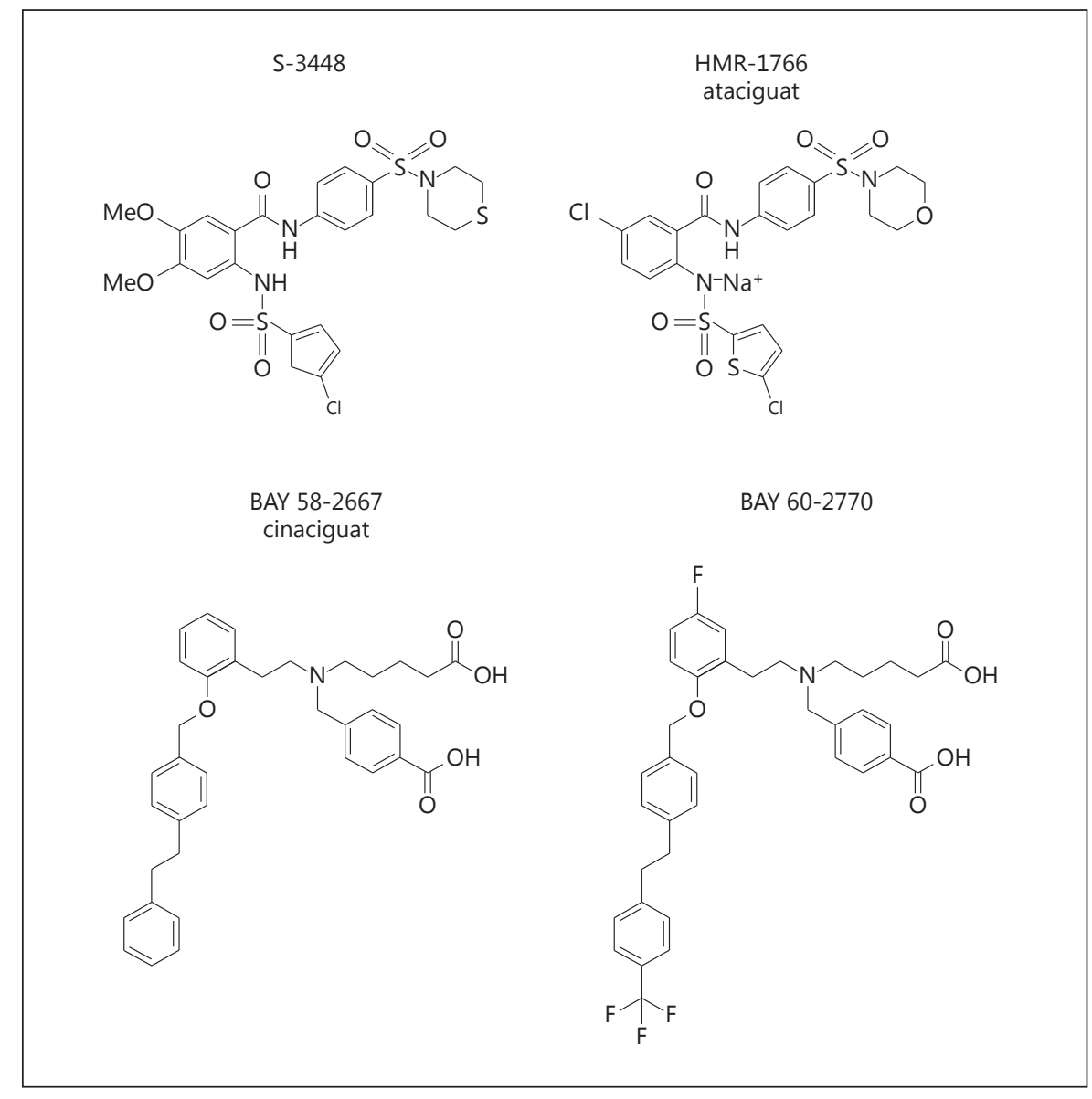

\section{Antifibrotic Effects of sGC Modulators}

\section{Attenuation of ECM Production in Kidney and Heart} Fibrosis

Fibrotic diseases are characterized by excessive accumulation of ECM [1]. Collagens represent the main structural proteins of ECM, and collagen content can be determined by histological visualization of collagen fibers by Sirius Red staining, immunostaining, or by detection of the collagen-specific amino acid hydroxyproline [27-29]. Additional markers that have been used to determine ECM accumulation in in vitro and in vivo preclinical studies include the ECM protein fibronectin (FN), expression of protease inhibitors that decrease matrix protein degradation, such as plasminogen activator inhibitor type 1 (PAI-1) and tissue inhibitors of matrix metalloproteinases (TIMPs), and matricellular proteins, such as osteopontin $[28,30]$.

The potential of sGC modulators to attenuate ECM accumulation has been studied in various models of fi- brotic kidney diseases. In a rat model of acute anti-Thy1induced mesangial proliferative glomerulonephritis that is characterized by intense ECM accumulation, treatment with the sGC stimulator BAY 41-2272 significantly reduced glomerular matrix accumulation, as determined by immunostaining for collagen IV and FN, compared with placebo on day 6 after disease induction [28]. In a model of chronic glomerulosclerosis that progresses autonomously from anti-Thyl-induced glomerulonephritis in uninephrectomized rats, BAY 41-2272 treatment significantly reduced ECM accumulation determined by histological examination 16 weeks after disease induction (after 15 weeks of treatment). Moreover, mRNA and protein expression of TGF $\beta 1, \mathrm{FN}$, and PAI-1 in tubulointerstitial tissue and protein expression of TGF $\beta 1$ and FN in glomerular tissue were significantly reduced by BAY 41-2272 treatment [31]. The sGC stimulator BAY 418543 enhanced renal recovery after relief of unilateral ureteral obstruction in a rat model, as determined by reduced matrix protein expansion, collagen IV deposition, 
and reduced mRNA expression of TGF $\beta 1, \mathrm{FN}$, and PAI1 [32].

The sGC activator cinaciguat exerted antifibrotic effects in a rat model of chronic kidney disease. Cinaciguat decreased glomerulosclerosis and renal perivascular and interstitial fibrosis, as determined by Sirius Red staining, but had no significant effect on cardiac fibrosis [33]. Likewise, the sGC stimulator riociguat reduced renal but not cardiac interstitial fibrosis in rats, but in contrast to cinaciguat, riociguat treatment did not affect glomerulosclerosis [34]. BAY 41-8543 treatment did not influence glomerulosclerosis or renal interstitial fibrosis but significantly attenuated uremic aortic remodeling involving reduced collagen I deposition and TIMP-1 gene expression [29].

In a high-renin model using hypertensive renin transgenic rats treated with the NOS inhibitor $\mathrm{N}^{\mathrm{G}}$-nitro-L-arginine methyl ester (L-NAME), riociguat significantly reduced glomerulosclerosis as well as renal and cardiac interstitial fibrosis, as determined by Sirius Red staining [34]. Consistently, riociguat significantly ameliorated glomerulosclerosis, renal and cardiac interstitial fibrosis, and renal but not cardiac perivascular fibrosis following salt-induced hypertension in salt-sensitive Dahl rats. In this animal model, riociguat additionally attenuated the increase in osteopontin, TIMP-1, and PAI-1 mRNA expression in the left ventricle and renal cortex, as well as urinary osteopontin, TIMP-1, and PAI-1 protein levels [30]. BAY 41-2272 significantly decreased interstitial and perivascular collagen accumulation, and TGF $\beta 1$ and type 1 collagen gene expression in the left ventricle in rats with hypertension induced by suprarenal aortic constriction [35]. In rats with severe angioproliferative $\mathrm{PAH}$ induced by hypoxia and the vascular endothelial growth factor antagonist SU5416, riociguat decreased collagen accumulation in the right ventricle [36]. Treatment with the sGC activator ataciguat reduced interstitial ECM accumulation in the noninfarcted left ventricle of rats with chronic heart failure after myocardial infarction, as determined by hydroxyproline content and Sirius Red staining. Moreover, ataciguat inhibited the induction of collagen expression in cardiac fibroblasts isolated from mice with heart failure and in primary human cardiac fibroblasts [27].

\section{Attenuation of ECM Production in Liver, Peritoneal and Skin Fibrosis}

Besides kidney and heart diseases, sGC modulators have demonstrated antifibrotic effects in other tissues. The sGC activator BAY 60-2770 attenuated liver fibrosis as determined by total hydroxyproline content and Sirius
Red staining in two rat models, the pig serum model, and the carbon tetrachloride model [37]. Furthermore, BAY 60-2770 treatment prevented progression and accelerated regression of thioacetamide-induced hepatic fibrosis in rats [38]. In an experimental model of peritoneal fibrosis induced by peritoneal injury in eNOS-deficient mice, treatment with BAY 41-2272 reduced ECM accumulation, as determined by type 1 collagen gene expression [39].

Treatment with BAY 41-2272 dose-dependently prevented development and induced regression of fibrosis, as determined by attenuated dermal thickening and ECM accumulation measured as dermal hydroxyproline content, in a mouse model of dermal fibrosis induced by intradermal injection of bleomycin and in the tight skin 1 mouse model [40]. Moreover, BAY 41-2272 attenuated dermal thickening and hydroxyproline content in a mouse model of dermal fibrosis induced by overexpression of constitutively active TGF $\beta$ receptor 1 and dosedependently reduced TGF $\beta 1$-induced type 1 collagen gene expression and total collagen content in human primary fibroblasts isolated from healthy individuals or patients with systemic sclerosis $[40,41]$. Similarly, riociguat dose-dependently reduced dermal thickness and hydroxyproline content in bleomycin-induced murine dermal fibrosis and the tight skin 1 model, and additionally attenuated dermal fibrosis as well as fibrosis of the small intestine in a mouse model of sclerodermatous chronic graft versus host disease [42].

Collectively, in vitro and in vivo data from model systems of tissue fibrosis demonstrated that application of sGC stimulators or activators uniformly attenuated ECM production and deposition - a hallmark of fibrosis - in various organs.

\section{Inhibition of Cellular Proliferation}

Effects of sGC modulators on cellular proliferation in fibrotic diseases can be studied either in in vitro proliferation assays using primary cells or cell lines or in situ by immunohistochemical detection of proliferating cells using specific antibodies against proliferation markers. The most commonly used cellular markers for proliferation are cell cycle-associated proteins such as $\mathrm{Ki}-67$ and proliferating cell nuclear antigen (PCNA) [43].

In vivo, treatment with the sGC stimulator BAY 412272 significantly reduced the number of PCNA-positive cells in the glomerulus of rats with anti-Thy1-induced mesangial proliferative glomerulonephritis, and double staining of PCNA with a CD90-specific antibody revealed that sGC stimulation in particular attenuated glomerular 
mesangial cell proliferation [28]. Similarly, in anti-Thy1induced chronic glomerulosclerosis in uninephrectomized rats, BAY 41-2272 markedly reduced proliferation of glomerular as well as tubulointerstitial cells [31]. Proliferation of the latter was also significantly reduced by the sGC stimulator BAY 41-8543 as determined by PCNA immunohistochemistry in a rat model of renal recovery after relief of unilateral ureteral obstruction [32].

BAY 41-8543 has additionally been shown to inhibit the proliferation of vascular smooth muscle cells induced by $5 / 6$ nephrectomy in male but not female rats, as determined by PCNA-positive cell numbers per aortic media cross-section [29]. Consistently, in vitro BAY 41-2272 significantly reduced proliferation of primary vascular smooth muscle cells isolated from thoracic aortas of male rats [44].

sGC stimulators have also been shown to exert antiproliferative effects in $\mathrm{PAH}$. While treatment with riociguat significantly inhibited the increase in PCNA-positive cells in the lung tissue of rats with hypoxia- and SU5416-induced severe angioproliferative PAH [36], stimulation with BAY 41-2272 significantly inhibited proliferation of human primary pulmonary arterial smooth muscle cells in vitro, probably via induction of PAI-2 [45].

Besides sGC stimulators, antiproliferative effects of the sGC activator cinaciguat have been investigated in cardiac fibroblasts. Cinaciguat significantly reduced basal proliferation of primary rat neonatal cardiac fibroblasts, as determined by $\mathrm{cDNA}$ synthesis measured by ${ }^{3} \mathrm{H}$-thymidine incorporation, but in contrast to BAY 41-2272, it did not reduce fetal calf serum-stimulated DNA synthesis [46]. Cinaciguat was consistently less effective than BAY 41-2272 in inducing expression of PAI-2, the putative mediator of the antiproliferative effects, in human primary pulmonary arterial smooth muscle cells [45].

Taken together, these findings indicate that the antiproliferative effect of cGMP enhancement by sGC modulators are more pronounced after sGC stimulation compared with activation.

\section{Inhibition and Reversal of TGF $\beta$-Induced \\ Myofibroblast Differentiation}

Besides enhanced proliferation and accumulation of excess ECM, fibrosis is characterized by the development and persistence of myofibroblasts. Myofibroblasts have a contractile phenotype and, in contrast to fibroblasts, express smooth muscle cell-specific proteins such as $\alpha$-smooth muscle cell actin (SMA) or calponin (CNN), which therefore serve as myofibroblast markers $[1,3,12]$.
TGF $\beta 1$-induced myofibroblast differentiation of fibroblasts is mediated by elevated expression of nicotinamide adenine dinucleotide phosphate oxidase 4 and concomitant downregulation of antioxidant enzymes such as SOD2 [1, 3]. Moreover, myofibroblasts are characterized by enhanced expression and secretion of a variety of growth factors and cytokines including FGF, TGF $\beta$, connective tissue growth factor, insulin-like growth factor (IGF) and modulators of the IGF signaling axis, e.g. IGFbinding protein 3 (IGFBP3) $[1,3,12]$.

sGC modulators have been shown to attenuate myofibroblast accumulation in various fibrosis models. Unilateral ureteral obstruction significantly increased the number of SMA-expressing tubulointerstitial cells in rat kidneys and the sGC stimulator BAY 41-8543 enhanced renal recovery after obstruction relief by reducing myofibroblast counts [32]. Similarly, BAY 41-2272 significantly decreased the number of SMA-stained cells in tissue sections from the left ventricle of rats with hypertension induced by suprarenal aortic constriction, and reduced SMA protein expression in cultured rat neonatal cardiac fibroblasts as determined by Western blotting [35]. In human cardiac fibroblasts, the sGC activator ataciguat suppressed the induction of SMA and connective tissue growth factor in response to TGF $\beta 1$ stimulation in vitro [27]. Moreover, ataciguat ameliorated the decrease in SOD2 protein expression in the noninfarcted left ventricle of rats with chronic heart failure after myocardial infarction [27].

The sGC activator BAY 60-2770 significantly attenuated myofibroblast activation as determined by SMA immunohistochemistry and Western blotting in thioacetamide-induced hepatic fibrosis in rats [38]. In addition, BAY 60-2770 reduced SMA expression in advanced hepatic fibrosis and inhibited myofibroblast activation of hepatic stellate cells in vitro [38]. BAY 41-2272 reduced myofibroblast accumulation in injury-induced peritoneal fibrosis in eNOS-deficient mice, as determined by SMA gene expression [39].

sGC stimulation by BAY 41-2272 or riociguat dosedependently reduced myofibroblast counts in murine dermal fibrosis induced by intradermal injection of bleomycin and in the tight skin 1 mouse model [40, 42]. Furthermore, sGC stimulation attenuated the numbers of SMA-expressing cells in a mouse model of dermal fibrosis induced by overexpression of constitutively active TGF $\beta$ receptor 1 and in a mouse model of sclerodermatous chronic graft versus host disease [41, 42].

In vitro, sGC stimulation by BAY 41-2272 or sGC activation by BAY 60-2770 inhibited and reversed TGF $\beta 1$ induced fibroblast-to-myofibroblast differentiation of 
primary human dermal fibroblasts and primary human prostatic stromal cells, as determined by SMA, CNN1, and IGFBP3 mRNA and protein levels [41, 47]. Similarly, in pulmonary fibroblasts, the sGC activator cinaciguat in combination with PDE5 inhibition attenuated myofibroblast differentiation [48].

Collectively, in vivo and in vitro data from fibrosis model systems demonstrate that sGC modulators inhibit myofibroblast activation and persistence in various organs. Inhibition of the activation of the primary effector cells of fibrosis appears to be a central mechanism of the observed antifibrotic properties of sGC stimulators and activators and is in line with data obtained after enhancement of the NO/cGMP signaling pathways by $\mathrm{NO}$ donors or PDE5 inhibitors $[1,3]$.

\section{Molecular Pathways Underlying Antifibrotic Effects of sGC Modulators}

Most of the above-detailed antifibrotic effects of sGC modulators are descriptive and lack further investigation of the underlying molecular pathways. However, the fact that the antifibrotic activity of sGC modulators can be mimicked or enhanced by NO donors, PDE5 inhibitors or cell-permeable cGMP analogues, as well as inhibited by PKG inhibitors, strongly indicates that the effects are primarily mediated via elevation of cGMP $[1,47]$.

PKG is a central downstream target of cGMP. Consistently, antiproliferative effects of cGMP on cardiac fibroblasts were mediated via PKG type I [49]. Similarly, PDE5 inhibition inhibited prostatic fibroblast proliferation as well as endothelin-1 or PDGF-induced cell migration of bladder myofibroblasts in a PKG-dependent way $[50,51]$. While in vascular smooth muscle cells BAY 41-2272-induced inhibition of migration was restored by PKG inhibition, antiproliferative effects of the sGC stimulator appeared to be partially mediated via protein kinase A activation [44].

In contrast to proliferation, the potential of PDE5 inhibition to block TGF $\beta$-induced myofibroblast differentiation of prostatic stromal cells was unaffected by PKG inhibition. However, inhibition of the MAPK/ERK (extracellular signal-regulated kinase) pathway restored the capacity of TGF $\beta$ to induce differentiation in the presence of a PDE5 inhibitor [50]. Of note, in dermal fibroblasts, stimulation of sGC by BAY 41-2272 also interfered with TGF $\beta$-dependent ERK signaling [41]. However, unlike inhibition of myofibroblast differentiation using PDE5 inhibitors, reversal of differentiation in prostatic stromal cells was neither affected by MAPK/ERK inhibition nor by PKG inhibition. In contrast, PDE5 inhibition in predifferentiated myofibroblasts resulted in an attenuation of PI3K/ AKT signaling. Consistently, PI3K or AKT inhibition mimicked the effects of cGMP enhancement and induced reversal of the cells to a fibroblast-like phenotype [14].

Brain natriuretic peptide, an activator of membranebound guanylate cyclases (pGC), decreased collagen synthesis and increased matrix metalloproteinases via PKG signaling in cardiac fibroblasts [52]. Atrial natriuretic peptide, an activator of pGC, or a cGMP analog inhibited TGF $\beta$-stimulated PAI-1 mRNA expression in cardiac fibroblasts, an effect that could be blocked by PKG inhibition [53]. Downstream of PKG, these effects appeared to be mediated via phosphorylation of Smad3 (at residues Ser309 and Thr388), thus preventing Smad3 nuclear translocation, suggesting a potential molecular mechanism that contributes to the antifibrotic effects of enhanced cGMP signaling [53]. Although pGC and sGC pathways seem highly compartmentalized in cells and tissues and might also be differentially regulated by PDEs, these results further support the broad antifibrotic potential of cGMP increase [54].

However, the detailed molecular mechanisms underlying the association of aging and fibrosis still remain elusive. Of note, AGEs that accumulate during aging, timeand dose-dependently decreased NO production, cGMP synthesis and PKG activation in rat renal fibroblasts [6]. Taken together, the above-mentioned impaired capacity to induce the NFE2-related factor 2 antioxidant response in cells from aged mice [10] suggests that aging makes cells/tissues more susceptible to a persistent pro-oxidant shift in redox homeostasis that further attenuates $\mathrm{NO}$ / cGMP signaling. Current knowledge on redox signaling and oxidative stress that promotes and perpetuates fibrosis in the context of aging has recently been reviewed elsewhere [11]. Future research will have to be performed to clarify the molecular alterations during aging and their relation to oxidative stress and inflammation, which are causative for higher risk of fibrotic diseases.

\section{Conclusions}

Fibrosis develops in response to tissue damage or inflammation via the action of growth factors secreted by activated platelets and infiltrating leukocytes, in particular TGF $\beta$. Aging is considered a risk factor for fibrosis that likely promotes fibrogenesis via various mechanisms such as elevated oxidative stress, cellular senescence or 


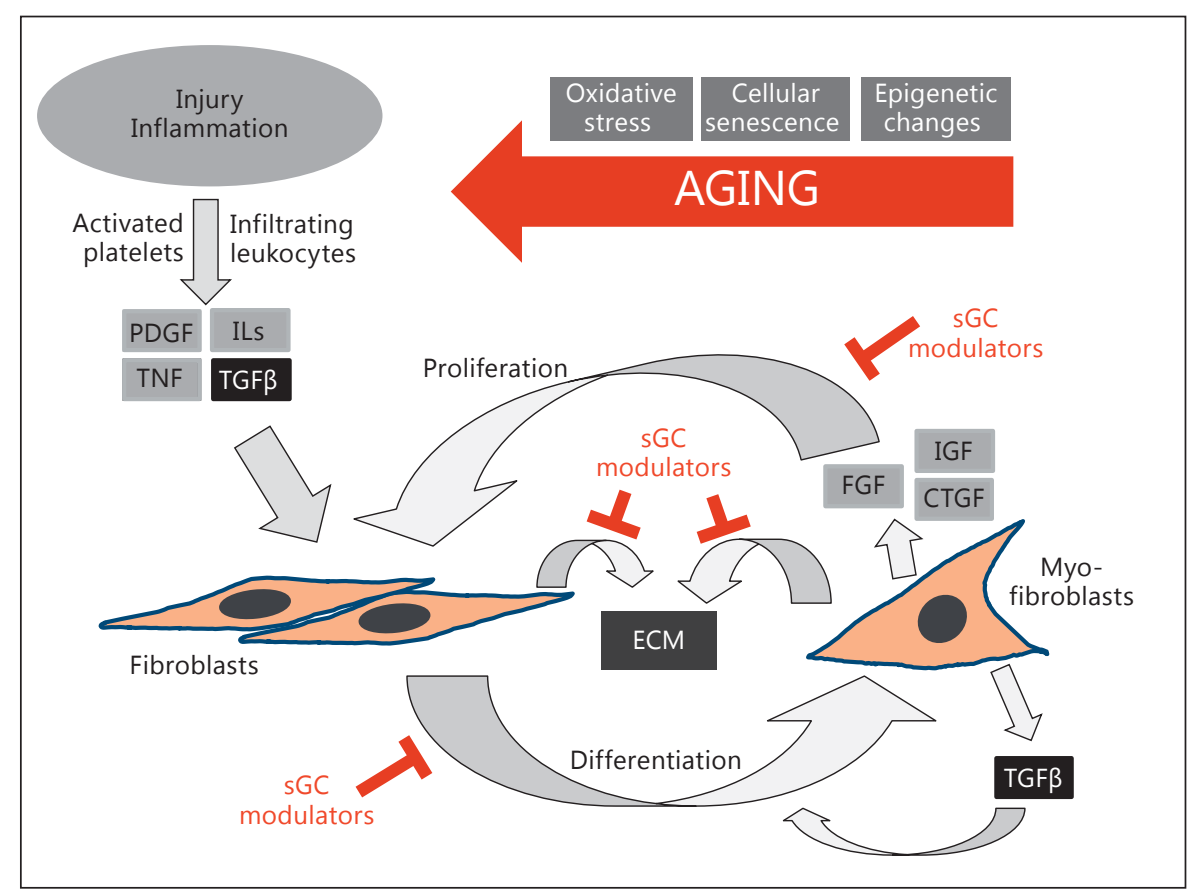

Fig. 4. Model of fibrogenesis and the antifibrotic effects mediated by sGC modulators. In response to tissue injury or inflammation, growth factors secreted by activated platelets and infiltrating leukocytes, in particular TGF $\beta$, induce fibroblast-to-myofibroblast differentiation and ECM deposition by local fibroblasts and myofibroblasts. Accumulating myofibroblasts in turn secrete factors that stimulate fibroblast proliferation as well as myofibroblast differentiation in auto- and paracrine feed-forward loops. Aging is considered a risk factor for fibrosis that likely promotes fibrogenesis via various mechanisms such as elevated oxidative stress, cellular senescence or epigenetic changes. Enhancement of $\mathrm{NO} /$
cGMP signaling by sGC stimulators or sGC activators has been shown to reduce ECM deposition, attenuate fibroblast proliferation as well as inhibit or even revert myofibroblast differentiation in vitro and in vivo, indicating that sGC modulators represent a promising therapeutic option for fibrotic diseases. cGMP, cyclic guanosine monophosphate; CTGF, connective tissue growth factor; ECM, extracellular matrix; FGF, fibroblast growth factor; IGF, insulin-like growth factor; IL, interleukin; NO, nitric oxide; PDGF, platelet-derived growth factor; sGC, soluble guanylate cyclase; TGF $\beta$, transforming growth factor- $\beta$; TNF, tumor necrosis factor. epigenetic changes. TGF $\beta$-dependent fibrosis is characterized by myofibroblast differentiation of local fibroblasts and ECM production and deposition by fibroblasts and myofibroblasts, respectively. Growth factors released by myofibroblasts, such as FGF, IGF, and TGF $\beta$, in turn stimulate proliferation as well as myofibroblast differentiation of the remaining fibroblast pool. Preclinical studies revealed that enhancement of $\mathrm{NO} / \mathrm{cGMP}$ signaling by sGC stimulators or sGC activators ameliorates the development of tissue fibrosis in various organs as demonstrated by reduced ECM deposition, attenuated proliferation, and reduced myofibroblast differentiation in vitro and in vivo (fig. 4). sGC modulators have been approved for the treatment of $\mathrm{PAH}$ and $\mathrm{CTEPH}$, and are currently under investigation for other indications, including heart failure and cystic fibrosis. Findings indicate that application of sGC modulators represents a promising therapeutic op- tion for fibrotic diseases that might at least slow disease progression. However, results from the ongoing clinical trials, e.g. in patients with systemic sclerosis, and future clinical studies are needed to prove the antifibrotic efficacy of these drugs.

\section{Acknowledgements}

The authors wish to cordially thank Drs. Markus Follmann and Michael Hahn, Medicinal Chemistry of Bayer Pharma AG at Wuppertal, for providing the molecular structures of sGC stimulators and of sGC activators.

\section{Disclosure Statement}

Peter Sandner is an employee of Bayer HealthCare. 


\section{References}

1 Sampson N, Berger P, Zenzmaier C: Redox signaling as a therapeutic target to inhibit myofibroblast activation in degenerative fibrotic disease. Biomed Res Int 2014;2014: 131737.

2 Wick G, Grundtman C, Mayerl C, Wimpissinger T, Feichtinger J, Zelger B, Sgonc R, Wolfram D: The immunology of fibrosis. Annu Rev Immunol 2013;31:107-135.

3 Sampson N, Berger P, Zenzmaier C: Therapeutic targeting of redox signaling in myofibroblast differentiation and age-related fibrotic disease. Oxid Med Cell Longev 2012; 2012:458276

4 Thannickal VJ, Zhou Y, Gaggar A, Duncan SR: Fibrosis: ultimate and proximate causes. J Clin Invest 2014;124:4673-4677.

5 Richter K, Kietzmann T: Reactive oxygen species and fibrosis: further evidence of a significant liaison. Cell Tissue Res DOI: 10.1007/ s00441-016-2445-3.

6 Huang J, Chuang L, Guh J, Chen C, Yang Y, Chiang T, Hung M, Liao T: Effect of nitric oxide-cGMP-dependent protein kinase activation on advanced glycation end-product-induced proliferation in renal fibroblasts. J Am Soc Nephrol 2005;16:2318-2329.

7 Begley LA, Kasina S, MacDonald J, Macoska JA: The inflammatory microenvironment of the aging prostate facilitates cellular proliferation and hypertrophy. Cytokine 2008;43:194199.

8 Vignozzi L, Gacci M, Cellai I, Morelli A, Maneschi E, Comeglio P, Santi R, Filippi S, Sebastianelli A, Nesi G, Serni S, Carini M, Maggi M: PDE5 inhibitors blunt inflammation in human BPH: a potential mechanism of action for PDE5 inhibitors in LUTS. Prostate 2013; 73:1391-1402.

9 Sueblinvong V, Neveu WA, Neujahr DC, Mills ST, Rojas M, Roman J, Guidot DM: Aging promotes pro-fibrotic matrix production and increases fibrocyte recruitment during acute lung injury. Adv Biosci Biotechnol 2014;5:19-30.

10 Hecker L, Logsdon NJ, Kurundkar D, Kurundkar A, Bernard K, Hock T, Meldrum E, Sanders YY, Thannickal VJ: Reversal of persistent fibrosis in aging by targeting Nox4Nrf2 redox imbalance. Sci Transl Med 2014;6: 231 ra47.

11 Kurundkar A, Thannickal VJ: Redox mechanisms in age-related lung fibrosis. Redox Biol 2016;9:67-76.

12 Hinz B, Phan SH, Thannickal VJ, Prunotto M, Desmouliere A, Varga J, De Wever O, Maree M, Gabbiani G: Recent developments in myofibroblast biology: paradigms for connective tissue remodeling. Am J Pathol 2012;180 1340-1355.

13 Lafyatis R: Transforming growth factor $\beta$ - at the centre of systemic sclerosis. Nat Rev Rheumatol 2014;10:706-719.
14 Zenzmaier C, Kern J, Sampson N, Heitz M, Plas E, Untergasser G, Berger P: Phosphodiesterase type 5 inhibition reverts prostate fibroblast-to-myofibroblast trans-differentiation. Endocrinology 2012;153:5546-5555.

15 Stasch J, Pacher P, Evgenov OV: Soluble guanylate cyclase as an emerging therapeutic target in cardiopulmonary disease. Circulation 2011;123:2263-2273.

16 Ghofrani H, D’Armini AM, Grimminger F, Hoeper MM, Jansa P, Kim NH, Mayer E, Simonneau G, Wilkins MR, Fritsch A, Neuser D, Weimann G, Wang C: Riociguat for the treatment of chronic thromboembolic pulmonary hypertension. N Engl J Med 2013; 369:319-329.

17 Ghofrani H, Galiè N, Grimminger F, Grünig E, Humbert M, Jing Z, Keogh AM, Langleben D, Kilama MO, Fritsch A, Neuser D, Rubin LJ: Riociguat for the treatment of pulmonary arterial hypertension. N Engl J Med 2013;369: 330-340.

18 Gheorghiade M, Greene SJ, Butler J, Filippatos G, Lam CSP, Maggioni AP, Ponikowski P, Shah SJ, Solomon SD, Kraigher-Krainer E, Samano ET, Müller K, Roessig L, Pieske B: Effect of vericiguat, a soluble guanylate cyclase stimulator, on natriuretic peptide levels in patients with worsening chronic heart failure and reduced ejection fraction: the SOCRATES-REDUCED randomized trial. JAMA 2015;314: 2251-2262.

19 Schmidt HH, Hofmann F, Stasch J: Handbook of Experimental Pharmacology 191. cGMP: generators, effectors and therapeutic implications. Preface. Handb Exp Pharmacol 2009; v-vi.

20 Stasch J, Hobbs AJ: NO-independent, haemdependent soluble guanylate cyclase stimulators. Handb Exp Pharmacol 2009;277-308.

21 Schmidt HH, Schmidt PM, Stasch J: NO- and haem-independent soluble guanylate cyclase activators. Handb Exp Pharmacol 2009;309339.

22 Mittendorf J, Weigand S, Alonso-Alija C, Bischoff E, Feurer A, Gerisch M, Kern A, Knorr A, Lang D, Muenter K, Radtke M, Schirok H, Schlemmer K, Stahl E, Straub A, Wunder F, Stasch J: Discovery of riociguat (BAY 63-2521): a potent, oral stimulator of soluble guanylate cyclase for the treatment of pulmonary hypertension. Chem Med Chem 2009;4: 853-865.

23 Follmann M, Griebenow N, Hahn MG, Hartung I, Mais F, Mittendorf J, Schäfer M, Schirok H, Stasch J, Stoll F, Straub A: The chemistry and biology of soluble guanylate cyclase stimulators and activators. Angew Chem Int Ed Engl 2013;52:9442-9462.
24 Pieske B, Butler J, Filippatos G, Lam C, Maggioni AP, Ponikowski P, Shah S, Solomon S, Kraigher-Krainer E, Samano ET, Scalise AV, Müller K, Roessig L, Gheorghiade M: Rationale and design of the SOluble guanylate $\mathrm{Cy}$ clase stimulatoR in heArT failurE Studies (SOCRATES). Eur J Heart Fail 2014;16:10261038.

25 Stasch J, Schlossmann J, Hocher B: Renal effects of soluble guanylate cyclase stimulators and activators: a review of the preclinical evidence. Curr Opin Pharmacol 2015;21:95-104.

26 Evgenov OV, Pacher P, Schmidt PM, Haskó G, Schmidt HH, Stasch J: NO-independent stimulators and activators of soluble guanylate cyclase: discovery and therapeutic potential. Nat Rev Drug Discov 2006;5:755-768.

27 Fraccarollo D, Galuppo P, Motschenbacher S, Ruetten H, Schäfer A, Bauersachs J: Soluble guanylyl cyclase activation improves progressive cardiac remodeling and failure after myocardial infarction. Cardioprotection over ACE inhibition. Basic Res Cardiol 2014;109: 421

28 Hohenstein B, Daniel C, Wagner A, Stasch J, Hugo C: Stimulation of soluble guanylyl cyclase inhibits mesangial cell proliferation and matrix accumulation in experimental glomerulonephritis. Am J Physiol Renal Physiol 2005;288:F685-F693.

29 Stancu B, Krämer S, Loof T, Mika A, Amann K, Neumayer H, Peters H: Soluble guanylate cyclase stimulator BAY 41-8543 and female sex ameliorate uremic aortic remodeling in a rat model of mild uremia. J Hypertens 2015; 33:1907-1920; discussion 1921.

30 Geschka S, Kretschmer A, Sharkovska Y, Evgenov OV, Lawrenz B, Hucke A, Hocher B, Stasch JP: Soluble guanylate cyclase stimulation prevents fibrotic tissue remodeling and improves survival in salt-sensitive Dahl rats. PLoS One 2011;6:e21853.

31 Wang Y, Krämer S, Loof T, Martini S, Kron S, Kawachi H, Shimizu F, Neumayer H, Peters $\mathrm{H}$ : Stimulation of soluble guanylate cyclase slows progression in anti-thyl-induced chronic glomerulosclerosis. Kidney Int 2005; 68:47-61

32 Wang-Rosenke Y, Mika A, Khadzhynov D, Loof T, Neumayer H, Peters H: Stimulation of soluble guanylate cyclase improves renal recovery after relief of unilateral ureteral obstruction. J Urol 2011;186:1142-1149.

33 Kalk P, Godes M, Relle K, Rothkegel C, Hucke A, Stasch J, Hocher B: NO-independent activation of soluble guanylate cyclase prevents disease progression in rats with 5/6 nephrectomy. Br J Pharmacol 2006;148:853-859.

34 Sharkovska Y, Kalk P, Lawrenz B, Godes M, Hoffmann LS, Wellkisch K, Geschka S, Relle K, Hocher B, Stasch JP: Nitric oxide-independent stimulation of soluble guanylate cyclase reduces organ damage in experimental lowrenin and high-renin models. J Hypertens 2010;28:1666-1675. 
35 Masuyama H, Tsuruda T, Sekita Y, Hatakeyama K, Imamura T, Kato J, Asada Y, Stasch JP, Kitamura K: Pressure-independent effects of pharmacological stimulation of soluble guanylate cyclase on fibrosis in pressure-overloaded rat heart. Hypertens Res 2009;32:597603.

36 Lang M, Kojonazarov B, Tian X, Kalymbetov A, Weissmann N, Grimminger F, Kretschmer A, Stasch J, Seeger W, Ghofrani HA, Schermuly RT: The soluble guanylate cyclase stimulator riociguat ameliorates pulmonary hypertension induced by hypoxia and SU5416 in rats. PLoS One 2012;7:e43433.

37 Knorr A, Hirth-Dietrich C, Alonso-Alija C, Harter M, Hahn M, Keim Y, Wunder F, Stasch JP: Nitric oxide-independent activation of soluble guanylate cyclase by BAY 602770 in experimental liver fibrosis. Arzneimittelforschung 2008;58:71-80.

38 Xie G, Wang X, Wang L, Wang L, Atkinson RD, Kanel GC, Gaarde WA, Deleve LD: Role of differentiation of liver sinusoidal endothelial cells in progression and regression of hepatic fibrosis in rats. Gastroenterology 2012; 142:918-927.e6.

39 Kadoya H, Satoh M, Nagasu H, Sasaki T, Kashihara N: Deficiency of endothelial nitric oxide signaling pathway exacerbates peritoneal fibrosis in mice. Clin Exp Nephrol 2015; 19:567-575.

40 Beyer C, Reich N, Schindler SC, Akhmetshina A, Dees C, Tomcik M, Hirth-Dietrich C, Degenfeld G von, Sandner P, Distler O, Schett G, Distler JH: Stimulation of soluble guanylate cyclase reduces experimental dermal fibrosis. Ann Rheum Dis 2012;71:1019-1026.

41 Beyer C, Zenzmaier C, Palumbo-Zerr K, Mancuso R, Distler A, Dees C, Zerr P, Huang J, Maier C, Pachowsky ML, Friebe A, Sandner P, Distler O, Schett G, Berger P, Distler, Jörg HW: Stimulation of the soluble guanylate cyclase (sGC) inhibits fibrosis by blocking noncanonical TGF $\beta$ signalling. Ann Rheum Dis 2015;74:1408-1416.
42 Dees C, Beyer C, Distler A, Soare A, Zhang Y, Palumbo-Zerr K, Distler O, Schett G, Sandner P, Distler, Jörg HW: Stimulators of soluble guanylate cyclase (sGC) inhibit experimental skin fibrosis of different aetiologies. Ann Rheum Dis 2015;74:1621-1625.

43 Iatropoulos MJ, Williams GM: Proliferation markers. Exp Toxicol Pathol 1996;48:175-181.

44 Joshi CN, Martin DN, Fox JC, Mendelev NN Brown TA, Tulis DA: The soluble guanylate cyclase stimulator BAY 41-2272 inhibits vascular smooth muscle growth through the cAMP-dependent protein kinase and cGMPdependent protein kinase pathways. J Pharmacol Exp Ther 2011;339:394-402.

45 Zhang S, Zou L, Yang T, Yang Y, Zhai Z, Xiao F, Wang C: The sGC activator inhibits the proliferation and migration, promotes the apoptosis of human pulmonary arterial smooth muscle cells via the up regulation of plasminogen activator inhibitor-2. Exp Cell Res 2015;332:278-287.

46 Irvine JC, Ganthavee V, Love JE, Alexander AE, Horowitz JD, Stasch J, Kemp-Harper BK, Ritchie RH: The soluble guanylyl cyclase activator bay 58-2667 selectively limits cardiomyocyte hypertrophy. PLoS One 2012;7: e44481.

47 Zenzmaier C, Kern J, Heitz M, Plas E, Zwerschke W, Mattesich M, Sandner P, Berger P: Activators and stimulators of soluble guanylate cyclase counteract myofibroblast differentiation of prostatic and dermal stromal cells. Exp Cell Res 2015;338:162-169.

48 Dunkern TR, Feurstein D, Rossi GA, Sabatini F, Hatzelmann A: Inhibition of TGF-beta induced lung fibroblast to myofibroblast conversion by phosphodiesterase inhibiting drugs and activators of soluble guanylyl cyclase. Eur J Pharmacol 2007;572:12-22.
49 Smolenski A, Schultess J, Danielewski O, Garcia Arguinzonis, Maisa I, Thalheimer P, Kneitz S, Walter U, Lohmann SM: Quantitative analysis of the cardiac fibroblast transcriptome-implications for NO/cGMP signaling. Genomics 2004;83:577-587.

50 Zenzmaier C, Sampson N, Pernkopf D, Plas E, Untergasser G, Berger P: Attenuated proliferation and trans-differentiation of prostatic stromal cells indicate suitability of phosphodiesterase type 5 inhibitors for prevention and treatment of benign prostatic hyperplasia. Endocrinology 2010;151:3975-3984.

51 Gacci M, Andersson K, Chapple C, Maggi M, Mirone V, Oelke M, Porst H, Roehrborn C, Stief C, Giuliano F: Latest evidence on the use of phosphodiesterase type 5 inhibitors for the treatment of lower urinary tract symptoms secondary to benign prostatic hyperplasia. Eur Urol 2016;70:124-133.

52 Tsuruda T, Boerrigter G, Huntley BK, Noser JA, Cataliotti A, Costello-Boerrigter LC, Chen $\mathrm{HH}$, Burnett JC: Brain natriuretic peptide is produced in cardiac fibroblasts and induces matrix metalloproteinases. Circ Res 2002;91: 1127-1134.

53 Li P, Wang D, Lucas J, Oparil S, Xing D, Cao $X$, Novak L, Renfrow MB, Chen YF: Atrial natriuretic peptide inhibits transforming growth factor beta-induced Smad signaling and myofibroblast transformation in mouse cardiac fibroblasts. Circ Res 2008;102:185-192.

54 Lee DI, Zhu G, Sasaki T, Cho G, Hamdani N, Holewinski R, Jo S, Danner T, Zhang M, Rainer PP, Bedja D, Kirk JA, Ranek MJ, Dostmann WR, Kwon C, Margulies KB, Van Eyk, Jennifer E, Paulus WJ, Takimoto E, Kass DA: Phosphodiesterase 9A controls nitric-oxideindependent cGMP and hypertrophic heart disease. Nature 2015;519:472-476. 